\title{
RhoGDI2 confers resistance to 5-fluorouracil in human gastric cancer cells
}

\author{
ZHONG ZHENG $^{1 *}$, XIANG-YI HE ${ }^{2 *}$, JIAN-FANG LI $^{3}$, BEI-QIN YU ${ }^{3}$, XUE-HUA CHEN ${ }^{3}$, JUN JI $^{3}$, \\ JIA-NIAN ZHANG ${ }^{3}$, QIN-LONG GU ${ }^{3}$, ZHENG-GANG ZHU ${ }^{3}$ and BING-YA LIU ${ }^{3}$ \\ ${ }^{1}$ Department of Oncology, Fudan University Shanghai Cancer Center, Shanghai Medical College, Fudan University; \\ ${ }^{2}$ Department of Gastroenterology; ${ }^{3}$ Department of Surgery, Shanghai Key Laboratory of Gastric Neoplasms, \\ Shanghai Institute of Digestive Surgery, Ruijin Hospital, Shanghai Jiaotong University School of Medicine,
}

Shanghai 200025, P.R. China

Received June 5, 2012; Accepted August 8, 2012

DOI: $10.3892 / \mathrm{ol} .2012 .949$

\begin{abstract}
Resistance to 5-fluorouracil (5-FU) in patients with gastric cancer is a serious therapeutic problem and major efforts are underway to understand the underlying mechanisms. We have previously identified RhoGDI2 as a contributor to 5-FU resistance in colon cancer cells using 2D electrophoresis and mass spectrometry and the current study aimed to further investigate this role. The expression of RhoGDI2 in seven gastric cancer cell lines was positively correlated with resistance to 5-FU. Lower 5-FU sensitivity of isolated tumor cells from patients with gastric cancer was also associated with higher RhoGDI2 expression. Ectopic expression of RhoGDI2 in gastric cancer cells increased the resistance to 5-FU and reverted low dose 5-FU-induced G2/M phase arrest without affecting the population of sub-G1 cells. Overall, these findings suggest that RhoGDI2 is associated with 5-FU resistance and is a potential therapeutic target for enhancing chemotherapy efficacy in gastric cancer.
\end{abstract}

\section{Introduction}

Gastric cancer is the fourth most common type of cancer and the second leading cause of cancer-related mortality in the world (1). Chemotherapy plays a significant role in the treatment of gastric cancer in adjuvant and advanced settings. 5-fluorouracil (5-FU) and its derivatives (e.g., S-1 and Xeloda) are significant, effective agents used to treat gastric cancer.

Correspondence to: Professor Bing-Ya Liu, Shanghai Key Laboratory of Gastric Neoplasms, Department of Surgery, Shanghai Institute of Digestive Surgery, Ruijin Hospital, Shanghai Jiaotong University School of Medicine, Shanghai 200025, P.R. China

E-mail: byliu@sjtu.edu.cn

*Contributed equally

Key words: RhoGDI2, 5-fluorouracil resistance, gastric cancer, cell cycle
However, the efficacy of chemotherapy for gastric cancer is limited due to insensitivity and the development of drug resistance.

To reveal the mechanisms underlying 5-FU resistance, the proteomic profiles of 5-FU-resistant and -sensitive colon cancer cells have been previously compared by 2-D gel electrophoresis. It was revealed that RhoGDI2 was upregulated in 5-FU-resistant colon cancer cells ( $\mathrm{LoV} / 5-\mathrm{FU})$ and that the knockdown of RhoGDI2 expression by transfection with RhoGDI2-specific siRNA significantly increased the sensitivity to 5-FU in LoVo/5-FU. These data suggest that RhoGDI2 confers resistance to 5-FU in colon cancer cells.

RhoGDI2 belongs to a family of Rho GTPase dissociate inhibitors (GDIs). GDIs are pivotal regulators of Rho GTPase function typified by forming a complex with Rho GTPase, modulating their nucleotide exchange and membrane association. Therefore, they play a significant role in regulating the actin cytoskeleton, cell polarity, microtubule dynamics, membrane transport pathways and transcription factor activity $(2,3)$. Unlike other members of the family (such as RhoGDI1 and RhoGDI3), RhoGDI2 is preferentially expressed in hematopoietic cells and appears to have a narrow selectivity and lower binding affinity for Rho GTPases (4). RhoGDI2 associates with and negatively regulates Rac1 and Rac3 in breast cancer cells, but not RhoA, Cdc42 and RhoC (5), whereas it positively regulates Rac1 in human bladder cancer cells (6). RhoGDI2 is also a substrate for caspases and becomes cleaved in various cell types during apoptosis. The significant role of RhoGDI2 in cancer has been previously noted by several lines of study. RhoGDI2 expression appears to be inversely correlated with an invasive capacity in bladder cancer cell lines (7). The reduced expression of RhoGDI2 protein was associated with a poor prognosis of patients with advanced bladder cancer (8). By contrast, RhoGDI2 is overexpressed in human breast cancer cell lines and increases cancer cell invasion and motility in vitro (9). Choi et al also revealed that RhoGDI2 expression is positively correlated with tumor progression and metastasis potential in gastric cancer (10). Therefore, exploring the role of RhoGDI2 in gastric cancer is likely to aid our understanding of how it contributes to 5-FU resistance. 


\section{Materials and methods}

Cell culture. The human gastric cancer cell lines SNU16, SNU-1, AGS and KATO-III were obtained from the American Type Culture Collection (Rockville, MD, USA). The human gastric cancer cell lines MKN-45, MKN-28 and SGC7901 were obtained from the Chinese Academy of Sciences Cell Bank of Type Culture Collection (Shanghai, China). All the gastric cancer cell lines were cultured in RPMI-1640 medium (Invitrogen, Carlsbad, CA, USA) supplemented with 10\% FBS (PAA Laboratories GmbH, Morningside, Queensland, Australia).

Patients and tissues. Human gastric cancer tissue arrays were obtained from Outdo Biotech (Shanghai, China) with 88 individual cases of gastric cancer and matched normal colon tissue. For the in vitro chemosensitivity test, 12 tumor specimens were obtained from patients during primary surgery from the Department of Surgery at Ruijin Hospital (Shanghai, China). All tissues were obtained from untreated patients following informed consent. The study was approved by the Ethics Committee of Ruijin Hospital, Shanghai JiaoTong University School of Medicine, Shanghai, China. Certain sections of the specimens were transfered directly into tissue culture medium for cell culture, while the others were fixed with formalin for the IHC test.

RhoGDI2-expressing plasmid. To generate a RhoGDI2 expression plasmid, the full-length coding region of RhoGDI2 cDNA was amplified using the primers RhoGDI2-forward 5'-CAT ACT CGA GCG GAC AGA GAC GTG AAG CAC-3' and RhoGDI2-reverse 3'-CAC TGG ATC CGA GTG ACA GGG TGG GAA AAG-5' (the restriction sites of $X h o \mathrm{I}$ and $\mathrm{BamHI}$ are underlined) and inserted into pIRES2EGFP (Clontech Laboratories, Mountain View, CA, USA) at $\mathrm{XhoI}$ and BamHI sites. MKN-45 cells were transfected with pIRES2-EGFP-RhoGDI2 or pIRES2-EGFP using Lipofectamine 2000 reagent (Invitrogen) according to the manufacturer's instructions. Stable plasmid-transfected clones were selected using $800 \mu \mathrm{g} / \mathrm{ml} \mathrm{G} 418$ (Invitrogen) for 2 weeks, isolated colonies were picked up with tips and the cells were further cultured in the presence of $400 \mu \mathrm{g} / \mathrm{ml} \mathrm{G} 418$. MKN-45 cells transfected with pIRES2-EGFP-RhoGDI2 were named MKN-45/RhoGDI2 cells. MKN-45 cells transfected with pIRES2-EGFP were named MKN-45/GFP cells.

In vitro cytotoxicity assay. The MTT assay was used to determine the relative sensitivity of cell lines to 5-FU (Xudonghaipu Pharmaceutical Co., Shanghai, China), as described previously (11). For cell lines, cells plated in 96-well microplates were cultured with growth medium or treated with serial dilutions of 5-FU for $72 \mathrm{~h}$. Viable cells were measured with MTT (Sigma, St. Louis, MO, USA) and the results were expressed relative to the absorbance of cells grown in the absence of the drug. IC50 values were calculated by nonlinear regression analysis from triplicate independent experiments.

For patient samples, tissues were minced by scissors into RPMI-1640 medium. The tumor cells were then incubated at $37^{\circ} \mathrm{C}$ for $30 \mathrm{~min}$ in an enzyme cocktail containing $0.02 \%$ deoxyribonuclease I (Sigma), $0.05 \%$ pronase (Calbiochem,
Dormstadt, Germany) and $0.02 \%$ collagenase. The tumor cell suspension $\left(5 \times 10^{5}\right.$ cells $\left./ \mathrm{ml}\right)$ was then strained through a $150 \mu \mathrm{m}$ stainless steel mesh. The cells were centrifuged at $1,000 \mathrm{rpm}$ for $5 \mathrm{~min}$ and, following rinsing twice, the gastric carcinoma cells were verified by $0.25 \%$ trypan blue dye exclusion (Sigma). The cell number was adjusted to $1 \times 10^{5}$ cells/ $\mathrm{ml}$ and a $180 \mu \mathrm{l}$ aliquot of the tumor cell suspension was plated into each well of a 96-well cell culture plate (Nunc Inc., Rochester, NY, USA), followed by the addition of $20 \mu \mathrm{l}$ of each dose of 5 -FU $(1 \mathrm{mg} / \mathrm{ml})$. The cells were then incubated at $37^{\circ} \mathrm{C}$ for $72 \mathrm{~h}$ in a $5 \% \mathrm{CO}_{2}$ incubator. The cells were washed with phosphate-buffered saline and subsequently $25 \mu 1$ of MTT ( $2 \mathrm{mg} / \mathrm{ml}$; Sigma) was added and measured as described previously (11). The tumor inhibition rate (IR) was calculated from the following equation: IR $(\%)=(1-\mathrm{T} / \mathrm{C}) \mathrm{x}$ 100 , where $\mathrm{T}=\mathrm{OD}_{540}$ of the treated cells and $\mathrm{C}=\mathrm{OD}_{540}$ of the control cells.

Western blot analysis. Western blot analysis was performed as previously described (12). Briefly, cell lysates were separated by SDS-PAGE and transferred to a PVDF membrane. The blot was then probed with anti-RhoGDI2 (LabVision, Fremont, CA, USA) with a dilution of 1:1,000, followed by an incubation with a horseradish peroxidase-conjugated secondary antibody. The signal was detected using enhanced chemiluminescence (Millipore, Billerica, MA, USA). The expression level was quantified using the Image J programme (NIH).

Immunohistochemical staining. Immunohistochemical staining was performed as described previously using a DAKO EnVision+ System HRP (13). RhoGDI2 polyclonal antibody from LabVision was applied at a 1:2,000 dilution overnight at $4^{\circ} \mathrm{C}$. Purified rabbit-IgG was used as an isotype control. The stained sections were reviewed by two independent observers (Z.Z. and X.Y.H.) who had no prior knowledge of the clinical pathological data of the patients. A scoring method was used as reported previously based on the evidence that the specimens clearly demonstrated varying degrees of staining intensity and percentage of cell staining (14). Briefly, strong-intensity staining was scored as 3 , moderate as 2 , weak as 1 and negative as 0 . For each intensity score, the percentage of cells with that score was estimated visually. A combined weighted score consisting of the sum of the percentage of cells staining at each intensity level was calculated for each sample. The immunolabelling was categorised as negative (score $>30$ ) or positive (score $\leq 30$ ) for all the tissues.

Cell cycle analysis. Cells were plated in triplicate in 6-well plates. After $24 \mathrm{~h}$, cells adherent to plates were exposed to 0.5 or $5 \mu \mathrm{g} / \mathrm{ml} 5$-FU for $72 \mathrm{~h}$. The percentage of viable cells was determined by counting trypan blue exclusive cells by a hemocytometer. For cycle analysis, cells were harvested, fixed in 70\% ice-cold ethanol and incubated with propidium iodide $(20 \mu \mathrm{g} / \mathrm{ml})$ and RNase A $(100 \mu \mathrm{g} / \mathrm{ml})$ for $1 \mathrm{~h}$. Propidium iodide-stained DNA content was then assessed using a FACSCalibur flow cytometer (BD Biosciences, Franklin Lakes, NJ, USA) and the percentage of cells in each phase of the cell cycle was analysed using Flowjo 8.0 (Tree Star, Ashland, OR, USA). 

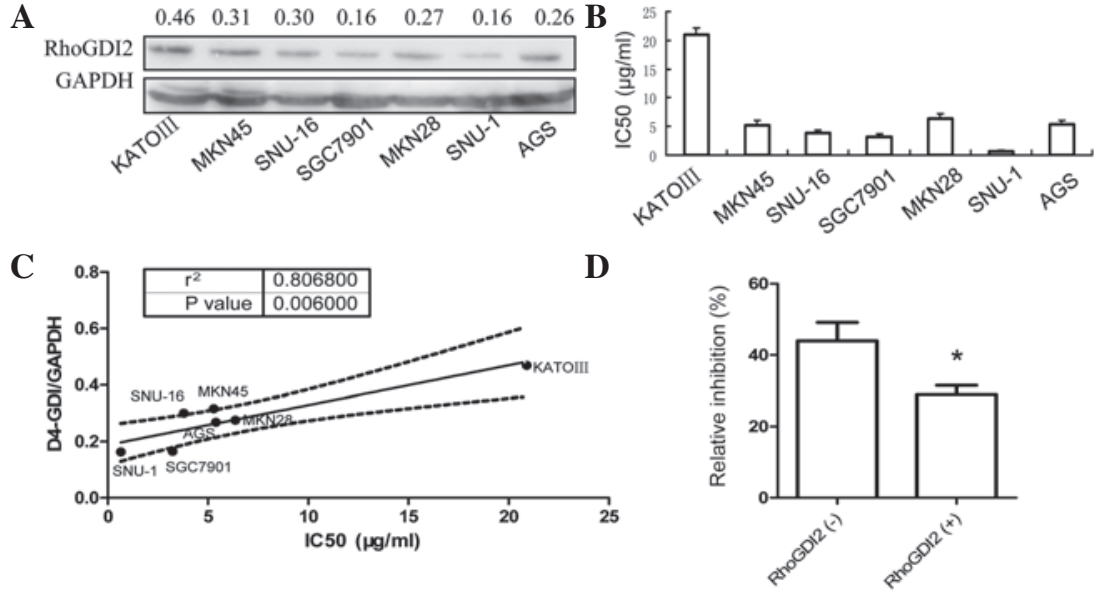

Figure 1. RhoGDI2 levels correlate with drug resistance in gastric cancer. (A) Western blotting analysis of RhoGDI2 expression in seven gastric cancer cell lines. (B) The sensitivity of gastric cancer cell lines to 5-FU was measured by MTT assay. (C) Pearson correlation of RhoGDI2 and IC50 of 5-FU in gastric cancer cell lines was analysed and plotted. (D) In vitro 5-FU sensitivity assay was performed in isolated tumor cells from patients with gastric cancer and RhoGDI2 expression in cancerous tissue was analysed by IHC, as described in Materials and methods. The inhibition rate (IR) in patients with positivelystained RhoGDI2 in cancerous cells was lower than that in negatively-stained cases ( $\mathrm{n}=6,{ }^{*} \mathrm{P}<0.05$ vs. RhoGDI2 negative). 5-FU, 5-fluorouracil.

A



B

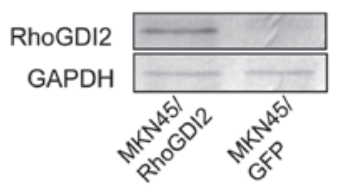

C


MKN-45/GFP + PBS

MKN-45/RhoGDI2 + PBS
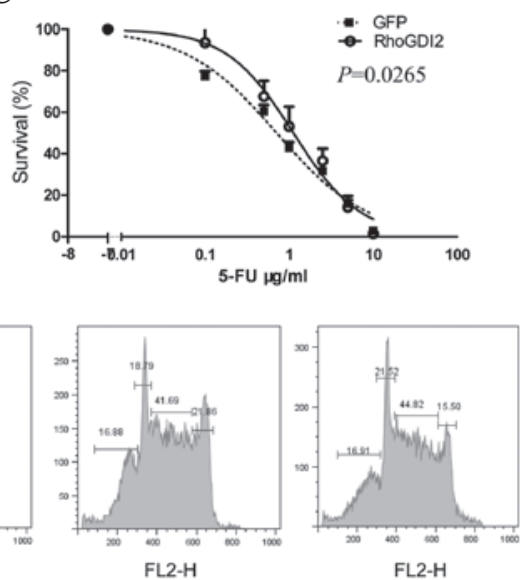

MKN-45/GFP + 5-FU

MKN-45/GFP + 5-FU

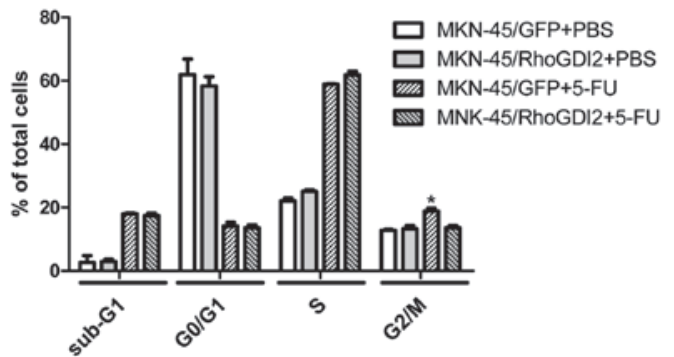

Figure 2. Ectopic expression of RhoGDI2 in gastric cancer increases resistance to 5-FU and reverts 5-FU-induced G2/M phase arrest. (A) Western blot analysis of RhoGDI2 expression. MKN-45 was stably transfected with the RhoGDI2 expression vector (MNK-45/RhoGDI2) or empty vector (MKN-45/GFP). GAPDH was used as a loading control. The immunoblot image was quantitated by densitometric analysis (right; $\mathrm{n}=3$ independent experiments). " $\mathrm{P}<0.05 \mathrm{vs}$. MKN-45/GFP. (B) 5-FU sensitivity of MKN-45/RhoGDI2 and MKN-45/GFP was measured by MTT assay (representative of three independent experiments; $\mathrm{P}<0.05$ vs. MNK-45/GFP). (C) SGC7901 cells were transiently transfected with pIRES2-RhoGDI2 or pIRES2-EGFP. Following $24 \mathrm{~h}$ of transfection, cells were collected and the 5-FU sensitivity was measured by MTT assay (representative of three independent experiments; P<0.05 vs. GFP). (D) MKN-45/RhoGDI2 and MKN-45/GFP were exposed to media containing $0.5 \mu \mathrm{g} / \mathrm{ml}$ or PBS for $72 \mathrm{~h}$. Cells were collected for cell cycle analysis by PI staining and flow cytometry. Data are expressed as mean percentage $\pm \mathrm{SD}$ of total cells from three independent experiments; " $\mathrm{P}<0.001 \mathrm{vs}$. MNK-45/GFP with 5-FU. 5-FU, 5-fluorouracil. 



C

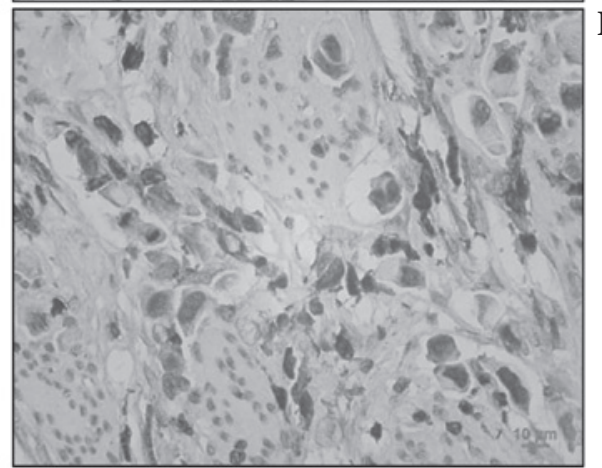

D

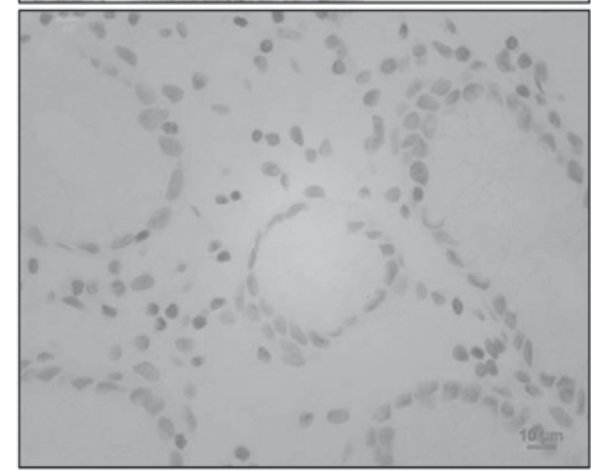

Figure 3. RhoGDI2 expression in gastrointestinal cancer. Gastrointestinal cancer tissues were immunostained with an antibody against RhoGDI2 (brown) and counterstained with hematoxylin (blue). (A) Normal gastric mucosa (x400); (B) gastric cancer (intestinal type; x400); (C) gastric cancer (diffuse type; x400); (D) negative staining control (x400).

Statistical analysis. Statistical analyses of data were performed using the Student's t-test or one-way analysis of variance, depending on the number of groups in comparison. Data that failed the test for normal distribution or homogeneous variance were analysed using the Mann-Whitney U or the Kruskal-Wallis tests. Correlations between RhoGDI2 and 5-FU sensitivity were calculated using the Pearson coefficient test. The statistical software SPSS version 14.0 (SPSS, Inc., Chicago, IL, USA) was used for analysis. $\mathrm{P}<0.05$ was considered to indicate a statistically significant result.

\section{Results}

Negative correlation between RhoGDI2 expression and 5-FU sensitivity in human gastric cancer. Western blot analysis was used to determine the level of RhoGDI2 in seven human gastric cancer cell lines. As shown in Fig. 1A, RhoGDI2 was expressed in all seven gastric cancer cell lines. The poorly differentiated cell line KATO-III derived from signet ring carcinoma had the highest level of RhoGDI2, which was 1.87-fold higher than that of the well-differentiated cell line, SGC7901. The sensitivity to 5-FU of each gastric cancer cell line was measured by the MTT assay and the IC50 values are indicated in Fig. 1B. Of note, the IC50 of each cell line positively correlates with the RhoGDI2 level ( $\mathrm{P}=0.006$, Fig. 1C). These results indicate that RhoGDI2 is associated with resistance to 5-FU in gastric cancer cells.

Subsequently, the 5-FU sensitivity of tumor cells isolated from gastric cancer tissues was tested and compared with the level of RhoGDI2 expression. Since the RhoGDI2 was markedly expressed in stroma, IHC was used to measure RhoGDI2 expression specifically in cancer cells. Consistent with the results obtained using the cell lines, the 5-FU sensitivity of patient cancer cells expressing RhoGDI2 was 30\% less than that of the negative controls $(\mathrm{P}<0.05$, Mann-Whitney $\mathrm{U}$ test $)$, as shown in Fig. 1D.

Ectopic expression of RhoGDI2 in gastric cancer cells induces resistance to 5-FU and reverts 5-FU induced G2/M phase arrest. To determine whether increased levels of RhoGDI2 induced 5-FU resistance, a gastric cancer cell line (MKN-45/RhoGDI2) was established that had been stably transfected with a RhoGDI2 expression vector. The RhoGDI2 level was markedly increased in MKN-45/RhoGDI2, compared with the control cell line MKN-45/GFP (Fig. 2A), while the sensitivity to 5-FU was decreased (Fig. 2B, P<0.0001). To verify that this effect was not a cell type-specific phenomenon, another gastric cancer cell line, SGC7901, was transiently transfected with the RhoGDI 2 coding vector. Following $24 \mathrm{~h}$ of transfection, cells were replated for the 5-FU sensitivity test. The IC50 of 5-FU in cells transfected with RhoGDI2 vector was increased by $60 \%$ compared with cells transfected with a GFP vector ( $\mathrm{P}=0.0265$, Fig. $2 \mathrm{C})$.

To determine whether RhoGDI2 influences the cell cycle following 5-FU treatment, PI staining followed by flow cytometry was used. It is reported that the dual antitumor effects of 5-FU on the cell cycle is dependent on 5-FU concentration: exposure to low dose 5-FU resulted in G2-phase arrest and mitotic catastrophe, whereas a high dose of 5-FU induced G1-phase arrest and apoptosis (15). Ectopic expression of RhoGDI2 alone did not influence the cell cycle distribution (Fig. 2D). Following $0.5 \mu \mathrm{g} / \mathrm{ml}$ (low dose) 5-FU treatment for $72 \mathrm{~h}$, the percentage of MKN-45/GFP cells in G1 decreased, whereas the proportion of cells in S and G2 phase increased. 
Compared with MKN-45/GFP cells treated with 5-FU, there were fewer MKN-45/RhoGDI2 cells in G2/M $(\mathrm{P}<0.05)$ following 5-FU treatment, but no difference was observed in PBS-treated MKN-45/RhoGDI2 cells. In addition, the sub-G1 proportion, known as apoptotic cells, remained unchanged between MKN-45/GFP and MKN-45/RhoGDI2 cells following exposure to 5-FU. Exposure to a high dose of 5-FU $(5 \mu \mathrm{g} / \mathrm{ml})$ resulted in G1/arrest in MKN-45/GFP and MKN-45/RhoGDI2 and there was no difference in sub-G1, G1, S, G2/M phase proportion between them (data not shown). These results suggest that RhoGDI2 reverts 5-FU-induced $\mathrm{G} 2 / \mathrm{M}$ phase arrest without influencing apoptosis and G1 phase arrest.

RhoGDI2 was upregulated in human gastric cancer. To determine the expression of RhoGDI2 in patients with gastric cancer, RhoGDI2 was analysed by IHC. As shown in Fig. 3, benign gastric epithelial cells were negatively or weakly (3 out of 88 cases) stained with RhoGDI2 antibody. By contrast, $53.4 \%$ gastric cancer specimens (47 out of 88 cases) were positively stained with anti-RhoGDI2 ( $\mathrm{P}<0.01$ vs. normal gastric mucosa). Thus, RhoGDI2 was overexpressed in gastric cancer cells. In intestinal-type gastric cancer cells, RhoGDI2 was mainly expressed in the plasma (Fig. 3B). However, diffuse-type gastric cancer cells were also markedly stained with anti-RhoGDI2 in the nucleus (Fig. 3C).

\section{Discussion}

In our previous study using 2D electrophoresis-mass spectrometry, it was revealed that RhoGDI2 is a contributor to 5-FU resistance in colon cancer (11). The current study demonstrated that RhoGDI2 also confers resistance to 5-FU in gastric cancer cells. Firstly, the endogenous level of RhoGDI2 was correlated with 5-FU resistance. Secondly, the ectopic expression of RhoGDI2 induced resistance to 5-FU. In addition, gastric cancer cells isolated from patients with higher levels of RhoGDI2 were more resistant to 5-FU. Other groups have shown that RhoGDI2 was overexpressed in chemotherapy-resistant fibrosarcoma cells and paclitaxel-resistant ovarian cancers, respectively $(16,17)$. However, Lee et al (18) recently revealed contradictory results suggesting that the chemo-resistance in ovarian serous carcinomas is associated with the downregulation of RhoGDI2 expression. Hee et al (19) reported that RhoGDI2 confers resistance against cisplatin-induced apoptosis in gastric cancer cells. The results of the current study lead to the conclusion that high levels of RhoGDI 2 expression are associated with chemotherapy resistance in certain types of cancers, including gastroenterological cancer.

The role of RhoGDI2 in apoptosis is contradictory. RhoGDI 2 is proteolysed by caspase- 3 during Fas-induced apoptosis (20) or in Burkitt-like lymphoma cells with taxol or epirubicin (21). However, the function of RhoGDI2 cleavage has only recently been elucidated. Based on the observation that the expression of a non-cleavable RhoGDI2 D19A mutant did not protect $\mathrm{CHO}$ cells against apoptosis induction, it was concluded that RhoGDI2 cleavage does not contribute to cell death (22). By contrast, another study suggested that the cleavage of RhoGDI2 by caspase-3 is not a functionally irrel- evant effect of caspase activation during apoptosis but rather expedites the progression of the apoptotic process (23). Unlike bladder cancer and lymphoma cells, RhoGDI 2 was reported as an oncogene in breast, colon and gastric cancer cells $(9,10,24)$. A Korean group reported that RhoGDI2 confers resistance to cisplatin-induced apoptosis in gastric cancer cells by upregulating Bcl-2 expression and activating PLC $\gamma(19,25)$. Our results also demonstrated that RhoGDI2 is involved in the resistance to 5-FU in gastric cancer cells. However, these data suggest that the resistance was not due to preventing apoptosis, but reverting G2/M phase arrest by RhoGDI2. RhoGDI2 may exert its chemo-resistant function through distinct mechanisms in response to various drugs, depending on the cell type.

It has been reported that there are dual antitumor effects of 5-FU on the cell cycle depending on 5-FU concentration: exposure to low dose 5-FU resulted in G2-phase arrest and mitotic catastrophe, whereas a high dose of 5-FU induced G1-phase arrest and apoptosis (15). In MKN-45 cells, similar dual effects were observed at 0.5 and $5 \mu \mathrm{g} / \mathrm{ml} \mathrm{5-FU,}$ respectively. However, RhoGDI2 only affected the low dose 5-FU-induced G2/M arrest, but not G1 arrest induced by high dose 5-FU. This is consistent with the results shown in Fig. 2B, which demonstrate that the resistance to 5-FU conferred by RhoGDI2 expression is mainly present at low doses of 5-FU and disappeared at higher doses of 5-FU.

In conclusion, it is suggested that RhoGDI 2 is aberrantly overexpressed and is also a direct contributor to the resistance to chemotherapeutic agents, including 5-FU, in gastric cancer cells. The potential mechanism involves inhibiting 5-FU-induced G2/M phase arrest. In this context, RhoGDI2 may be used as a molecular target for the sensitisation of gastrointestinal cancer cells during chemotherapy and as a predictor of chemotherapy treatment outcomes.

\section{References}

1. Jemal A, Bray F, Center MM, Ferlay J, Ward E and Forman D: Global cancer statistics. CA Cancer J Clin 61: 69-90, 2011.

2. Heasman SJ and Ridley AJ: Mammalian Rho GTPases: new insights into their functions from in vivo studies. Nat Rev Mol Cell Biol 9: 690-701, 2008.

3. Jaffe $A B$ and Hall A: Rho GTPases: biochemistry and biology. Annu Rev Cell Dev Biol 21: 247-269, 2005.

4. Dovas A and Couchman JR: RhoGDI: multiple functions in the regulation of Rho family GTPase activities. Biochem J 390: 1-9, 2005.

5. Zhang Y, Rivera Rosado LA, Moon SY and Zhang B: Silencing of D4-GDI inhibits growth and invasive behavior in MDA-MB-231 cells by activation of Rac-dependent p38 and JNK signaling. J Biol Chem 284: 12956-12965, 2009.

6. Moissoglu K, McRoberts KS, Meier JA, Theodorescu D and Schwartz MA: Rho GDP dissociation inhibitor 2 suppresses metastasis via unconventional regulation of RhoGTPases. Cancer Res 69: 2838-2844, 2009.

7. Gildea JJ, Seraj MJ, Oxford G, et al: RhoGDI2 is an invasion and metastasis suppressor gene in human cancer. Cancer Res 62: 6418-6423, 2002.

8. Theodorescu D, Sapinoso LM, Conaway MR, Oxford G, Hampton GM and Frierson HF Jr: Reduced expression of metastasis suppressor RhoGDI2 is associated with decreased survival for patients with bladder cancer. Clin Cancer Res 10: 3800-3806, 2004.

9. Zhang Y and Zhang B: D4-GDI, a Rho GTPase regulator, promotes breast cancer cell invasiveness. Cancer Res 66: 5592-5598, 2006

10. Cho HJ, Baek KE, Park SM, et al: RhoGDI2 expression is associated with tumor growth and malignant progression of gastric cancer. Clin Cancer Res 15: 2612-2619, 2009. 
11. Zheng Z, Li J, He X, et al: Involvement of RhoGDI2 in the resistance of colon cancer cells to 5 -fluorouracil. Hepatogastroenterology 57: 1106-1112, 2010.

12. Wang YW, Qu Y, Li JF, et al: In vitro and in vivo evidence of metallopanstimulin-1 in gastric cancer progression and tumorigenicity. Clin Cancer Res 12: 4965-4973, 2006.

13. Yuen HF, Chan YP, Chan KK, et al: Id-1 and Id-2 are markers for metastasis and prognosis in oesophageal squamous cell carcinoma. Br J Cancer 97: 1409-1415, 2007.

14. Ben QW, Wang JC, Liu J, et al: Positive expression of L1-CAM is associated with perineural invasion and poor outcome in pancreatic ductal adenocarcinoma. Ann Surg Oncol 17: 2213-2221, 2010

15. Yoshikawa R, Kusunoki M, Yanagi H, et al: Dual antitumor effects of 5-fluorouracil on the cell cycle in colorectal carcinoma cells: a novel target mechanism concept for pharmacokinetic modulating chemotherapy. Cancer Res 61: 1029-1037, 2001.

16. Sinha P, Hütter G, Köttgen E, Dietel M, Schadendorf D and Lage H: Search for novel proteins involved in the development of chemoresistance in colorectal cancer and fibrosarcoma cells in vitro using two-dimensional electrophoresis, mass spectrometry and microsequencing. Electrophoresis 20: 2961-2969, 1999.

17. Goto T, Takano M, Sakamoto M, et al: Gene expression profiles with cDNA microarray reveal RhoGDI as a predictive marker for paclitaxel resistance in ovarian cancers. Oncol Rep 15: 1265-1271, 2006.

18. Lee DH, Chung K, Song JA, et al: Proteomic identification of paclitaxel-resistance associated hnRNP A2 and GDI 2 proteins in human ovarian cancer cells. J Proteome Res 9: 5668-5676, 2010.
19. Cho HJ, Baek KE, Park SM, et al: RhoGDI2 confers gastric cancer cells resistance against cisplatin-induced apoptosis by upregulation of Bcl-2 expression. Cancer Lett 311: 48-56, 2011.

20. Na S, Chuang TH, Cunningham A, et al: D4-GDI, a substrate of CPP32, is proteolyzed during Fas-induced apoptosis. J Biol Chem 271: 11209-11213, 1996.

21. Essmann F, Wieder T, Otto A, Müller EC, Dörken B and Daniel PT: GDP dissociation inhibitor D4-GDI (Rho-GDI 2), but not the homologous rho-GDI 1, is cleaved by caspase-3 during drug-induced apoptosis. Biochem J 346: 777-783, 2000.

22. Krieser RJ and Eastman A: Cleavage and nuclear translocation of the caspase 3 substrate Rho GDP-dissociation inhibitor, D4-GDI, during apoptosis. Cell Death Differ 6: 412-419, 1999.

23. Choi MR, Groot M and Drexler HC: Functional implications of caspase-mediated RhoGDI2 processing during apoptosis of HL60 and K562 leukemia cells. Apoptosis 12: 2025-2035, 2007.

24. Li X, Wang J, Zhang X, Zeng Y, Liang L and Ding Y: Overexpression of RhoGDI2 correlates with tumor progression and poor prognosis in colorectal carcinoma. Ann Surg Oncol 19: 145-153, 2012.

25. Cho HJ, Baek KE, Nam IK, et al: PLCgamma is required for RhoGDI2-mediated cisplatin resistance in gastric cancer. Biochem Biophys Res Commun 414: 575-580, 2011. 